\title{
A variable compliance, soft gripper
}

\author{
M. E. Giannaccini - I. Georgilas - I. Horsfield • \\ B. H. P. M. Peiris - A. Lenz - A. G. Pipe • \\ S. Dogramadzi
}

\begin{abstract}
Autonomous grasping is an important but challenging task and has therefore been intensively addressed by the robotics community. One of the important issues is the ability of the grasping device to accommodate varying object shapes in order to form a stable, multi-point grasp. Particularly in the human environment, where robots are faced with a vast set of objects varying in shape and size, a versatile grasping device is highly desirable. Solutions to this problem have often involved discrete continuum structures that typically comprise of compliant sections interconnected with mechanically rigid parts. Such devices require a more complex control and planning of the grasping action than intrinsically compliant structures which passively adapt to complex shapes objects. In this paper, we present a low-cost, soft cable-driven gripper, featuring no stiff sections, which is able to adapt to a wide range of objects due to its entirely soft structure. Its versatility is demonstrated in several experiments. In addition, we also show how its compliance can be passively varied to ensure a compliant but also stable and safe grasp.
\end{abstract}

Keywords Grasping - Soft robotics - Continuum robot · Variable compliance $\cdot$ Shape invariant grasping

\section{Introduction}

In the rather large research area of autonomous grasping we are focussing on the design of the physical manipulator. A

Electronic supplementary material The online version of this article (doi:10.1007/s10514-013-9374-8) contains supplementary material, which is available to authorized users.

M. E. Giannaccini (凶) · I. Georgilas · I. Horsfield · B. H. P. M. Peiris · A. Lenz · A. G. Pipe $\cdot$ S. Dogramadzi

Bristol Robotics Laboratory, Bristol BS16 1QY, UK

e-mail: maria.elena.giannaccini@brl.ac.uk novel design of a highly compliant gripper with variable stiffness was formulated. Its initial high compliance is exploited to passively adapt to the shape of the objects.

For robotic systems to grasp in human environments, the ability to address the uncertainty of the setting and the objects in it is of an utmost importance. For this to be achieved, a stable grasp must be realised under the ambiguity of the particular geometry of the object. Thus, it is important for the robotic end-effector to conform to the shape of the object, increasing the contact area and creating multiple contact points. This results in better stability and smaller forces between the end-effector and the object during manipulation. For this reason, initial end-effector compliance when forming the grasp is desirable. Compliance permits the gripper to conform its surfaces to those of the object without needing explicit control and sensing, a process called shape match by Deimel and Brock (2013) and Eppner et al. (2012). However, once the grasp is established, an end-effector must be stiff enough to impress sufficient force on the object surface to make the holding and lifting of the object possible. Due to the very high initial compliance of our gripper, this must be increased to a level which allows force transmission to other objects. Thus, in the final phase of the grasping, the gripper needs to be stiffer than it is in its completely compliant initial stage but it is always relatively compliant compared to stiff structures such as, for example, metal ones.

One approach to achieve stable grasping is to utilise robotic end-effectors that have some degree of anthropomorphic structure, composed of two or more digits with as many as 20 degrees of freedom (DOF) (SHADOW 2013) in total, the control of which could be rather complicated.

A good example of this type of end-effector is the highly anthropomorphic DLR hand arm system (Grebenstein et al. 2011) with 19 DOF in the hand and kinematics similar to the human on a functional basis. Another example of a state-of- 
the-art anthropomorphic robot hand is the Shadow Dexterous Hand ${ }^{\circledR}$. This 24-joint-hand is actuated by pneumatic muscles which provide 20 DOF (SHADOW 2013). Anthropomorphic robotic hands aim to replicate human hands, which is considered beneficial for applications like tele-operation. However, the physical structure and control of anthropomorphic robot hands are remarkably complex and demanding. In addition, humanoid hands sometimes feature rigid digits, which can be negative for compliance to the object shape. On the contrary, overall, the soft structure is able to encircle objects more compliantly than any internally rigid ones. In fact, the soft structure has infinite points of curvature while humanoid hands can only bend at the joints between rigid parts. The latter type of end-effectors also need multi-joint grasp planning and are typically costly to produce due their complex construction.

An alternative to this approach are simple, nonanthropomorphic gripper designs based on the principle of intelligence embodied in materials and shape (Pfeifer et al. 2007). These non-humanoid end-effector designs rely more on material properties and less on control and sensors. Good examples of these devices are the University of Chicago universal gripper and the Festo fin ray effect gripper, which are described in the next paragraphs. The two approaches mentioned above are both well established in grasping research, each with their strengths and weaknesses.

Grasping devices can also be divided into structures with either unidirectional or omnidirectional compliance. Such distinction characterises a purely physical and morphological issue. The unidirectional compliance is descriptive of a physical structure that will passively comply to an object shape only along a single direction. On the contrary, an omnidirectional compliant object will passively and compliantly modify its shape when impacted from any direction. Current omnidirectional compliant state of the art grippers have various degrees of physical shape adaptability but none is exploiting this characteristic to obtain functional omnidirectional compliance. This characteristic would ensure that every direction of the passive compliance is exploited to aid the grasping action.

Examples of unidirectional compliance can be found in the adaptive fin ray effect gripper implemented by Festo and the Fraunhofer Institute, the SDM hand and Hirose's soft manipulator. The first one exploits a parallel linkage to passively obtain a lifting motion of the object while it is being grasped (FESTO 2013). However, the linkage prevents a compliant behaviour in all directions other than the one intended for grasping. As previously argued, compliance and passive adaptability are desirable when dealing with the uncertainty of the unstructured environment. These characteristics are at the core of the design of the adaptive SDM hand by Dollar and Howe (2010). This under-actuated cable driven device is able to successfully grasp objects of various shapes, sizes and masses in multiple orientations. Hirose's (1978) soft manipulator was the first gripper to adapt itself passively to the grasped object shape. The drawback of these three devices is that their digit structures feature compliance only in one direction, hindering the passive shape-adaptation in non-compliant directions. However, the definition of unidirectionally compliant grippers comprises also the aforementioned anthropomorphic hands, since they feature passive compliance in the directions of movements allowed by their degrees of freedom.

Among the non-anthropomorphic grasping devices with omnidirectional compliance, the most relevant examples are the Whiteside group starfish-like gripper and the RBO hand. The first one features fingers that are soft actuators made of elastomer composites with internal air channels. Their lack of rigid structures and the intrinsic flexibility of the elastomer material makes them pliable structures, thus they feature omnidirectional compliance (Ilievski et al. 2011). The same working principle is used in the RBO hand, created in the Robotics and Biology Laboratory, Berlin (RBO hand. Deimel, Brock 2013). The compliant nature of these grippers and their elastic surfaces ensures a good shape match with the object but makes the fitting of sensors on their surface challenging.

The other gripper that successfully utilises omnidirectional compliance is the University of Chicago universal gripper (Brown et al. 2010). This device employs the variable compliance principle, the change of the system's compliance during operation. Such process is exploited in grasping, since it can passively conform to the object shape and then stiffen using material jamming. The same working mechanism has been exploited for a serial manipulator (Cheng et al. 2012).

Devices like Clemson's Octarm (McMahan et al. 2006) and the robot octopus arm of the Scuola Superiore Sant'Anna (SSSA) (Calisti et al. 2010) inspired our work by challenging the technical limits of soft robotics. The intended application of these mechanisms is different to the grasping task in human environments under uncertainty. For instance, the first robot is well suited to grasping large objects but unsuitable for small objects present in a home environment. The SSSA arm was a major source of inspiration for our device, which maintains a similar cable structure. Nonetheless, our device's purpose is to aptly grasp everyday objects in the context of a human-robot interaction, whilst SSSA's robotic arm is part of a biomimetic effort to reproduce the structure and function of an Octopus vulgaris arm in an aqueous environment.

\subsection{Our previous work and its application domain}

The material jamming method was used as a starting point in our system development for a device grasping an object from its side (Giannaccini et al. 2011). However, it was observed 
that in the lateral grasp, stiffening produces a shrinking effect in the radial direction, unfavourable to the grasping stability. For this reason, we decided to change the working principle of our gripper. Material jamming was discarded in favour of passive variable compliance. However, we retained the omnidirectional compliance, in order to achieve large contact area and stable grasp.

To this end, we utilise a passive variable compliance continuum structure. Our structure features passive variable compliance since there is no active control of its stiffness but the latter increases once the gripper is activated. It is a soft structure since it contains no rigid joints and it is fully flexible, unlike structures like the Elephant's Trunk robot arm by Clemson, which also features rigid structures (Hannan and Walker 2003).

The inspiration of our design came from invertebrates with hydrostatic skeletons, fluid filled cavities surrounded by muscle that, by contracting, can vary the structure's stiffness (Taylor and Kier 2003). Our present cable-driven system aims to replicate an omnidirectional, compliant and continuum structure, allowing it to be intrinsically adaptive to the shape of the object.

Variable compliance is extremely important for our grasping principle. In the beginning of the grasp, a high level of gripper compliance is guaranteed, in order to adapt its shape to the object. At the end of the gripper action, it is necessary to increase the gripper's stiffness to ensure a stable grasp. The gripper has a high degree of compliance to conform adequately to the object shape and then its structure must become stiffer to retain its grasp on the object.

Our research goal is to develop a device that can stably grasp and hold objects without tilting them, while mounted on a soft arm, apt for human-robot interaction, where position accuracy is hard to obtain (INTRO 2013). For this reason, a greater workspace, variable compliance and compliance to object geometry is needed to realise stable grasping for manipulation under the aforementioned uncertain environment. The large workspace of the gripper is also needed in order to compensate for the high uncertainty of object localisation systems. Our gripper only addresses the grasping of certain forms (a height of at least $50 \mathrm{~mm}$ is necessary) but these forms can display a great variability in the local shape. In order to pick up flat objects like a plate it would be necessary to replicate the design of this gripper and assemble them in a gripper which is capable of opposition. In that respect, the current gripper, in some ways, correlates to a single finger. The strength of this gripper, however, is on the simplicity of its control compared to other grippers, e.g. the humanoid hands which have a high dexterity but also a high control complexity because of their redundancy.

This paper is organised as follows: in Sect. 2, we describe how bioinspiration has led us to develop the variable compliance of our structure, its materials and methods and the two gripper versions. The gripper's planar trajectory and its variable compliance are described and are tested in Sect. 3. In the same section, it is also shown that the initial compliance permits multiple points of contact with the object. Furthermore, experiments which test both grasping and holding on a surface without tilting and grasping in free space (with the gripper supporting its own weight) are described in Sect. 4. In Sect. 5 the results of our work is summarised and discussed, the paper concludes (Sect. 6) with a brief outline of future work.

\section{Hardware implementation}

In this section the description of the hardware, shown in Fig. 1, for the proposed gripper is presented. The inspiration from nature, hydrostatic skeletons, is described and our engineering implementation is analysed. The design of the system and the materials used are explained and the two different variants of the prototype detailed.

\subsection{Bioinspiration}

Numerous invertebrates possess a hydrostatic skeleton, e.g. snails, caterpillars, earthworms and starfish. Animals featuring hydrostatic skeletons have a flexible body with few constraints on their degrees of freedom. Such characteristics are granted by their structure, an incompressible fluid filling an internal cavity surrounded by a flexible container. Typically this container comprises the body wall provided with muscles (Kier 2012). Hydrostatic skeletons muscles are arranged so that both the diameter and the length of their body can be actively controlled. The muscles that control these displacements are oriented in two directions. Longitudinal muscles

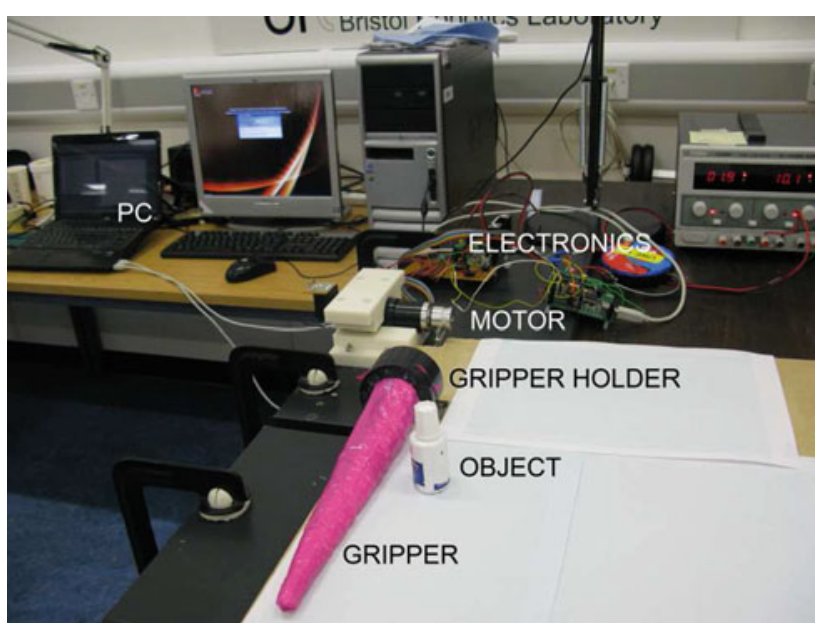

Fig. 1 Photograph of the continuum gripper (non actuated) on the testbench 
are orientated parallel to the long axis of their structure and control of the length of the structure. The muscle fibres that control the diameter dimension are all perpendicular to the long axis but they can, depending on the animal, have three arrangements: circular musculature, radial musculature and transverse musculature. Among the typical movements of invertebrates with hydrostatic skeletons there are: elongation, shortening, bending and stiffening. Invertebrates have inspired robotics research in the past; walking on land (Trimmer et al. 2006) or in water (Vaidyanathan et al. 2000) and crawling (Menciassi et al. 2006) are among the important tasks addressed with this approach. Our work, to the best of our knowledge, is the first to employ hydrostatic skeletons as inspiration for a grasping device.

The flexibility and the absence of rigid elements of invertebrate bodies inspired the soft structure and the lack of discrete joints /links of our device. Quasi-longitudinal cables on one side provide the bending movement in a similar manner to the longitudinal muscles in hydrostatic skeletons. Our device is inspired by the principle of the bending and stiffening movements of hydrostatic skeletons. The flexible material of our gripper's outer shell operates as the connective tissue, which passively controls the various dimensions of the hydrostatic skeleton (Kier 2012). However, it is very important to state that our aim is to design a bioinspired device, which takes inspiration from hydrostatic skeletons to tackle a practical grasping issue, and does not have as a first objective to reproduce as much as feasibly possible the biological specimen structure, as it is the case in biomimetic robotics.

\subsection{Design, materials and control}

The gripper's simple and inexpensive design consists of a flexible outer shell, shown in Fig. 2, filled with incompressible liquid and actuated by a pair of cables. Grasping action, in the horizontal plane (Giannaccini et al. 2012), and variable compliance is achieved simultaneously by pulling the cables. The outer shell is of conical shape and made of polyethylene, a flexible material. This material's textile strength is such that it does not expand when it is filled with fluid but, on the other hand, it is flexible enough to fold and allow a concertina. The system consisting of two $0.3 \mathrm{~mm}$ diameter Dyneema ${ }^{\circledR}$ cables (Fireline, Berkley), located at a $2.5^{\circ}$ angle from the longitu-

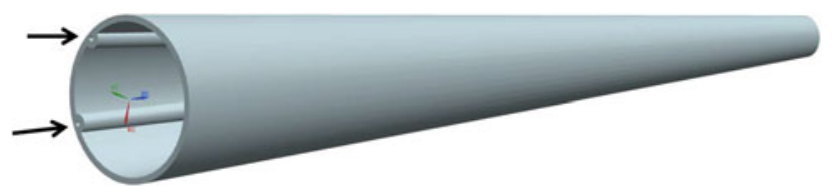

Fig. 2 UGS NX6 software model of the outer empty shell with silicone tubes. The two tubes and the cables inside them are on one side of the gripper. The two arrows point to the location of the cables dinal axis, due to the gripper's shape. They are lodged inside a $1.5 \mathrm{~mm}$ in diameter flexible silicone tube (Silex) attached to the shell with silicone paste (Soudal). Two configurations of the gripper's cable system have been designed, exhibiting different grasping behaviours, which are analysed in detail in the following section. The gripper is $250 \mathrm{~mm}$ long; with a maximum diameter (at the base) of $40 \mathrm{~mm}$ and minimum diameter (at the tip) of $15 \mathrm{~mm} .87 \%$ of the internal volume of the gripper is filled with degassed water, then the air filling the rest of the volume is vacuumed out. Further information on the system design is available in previous work (Giannaccini et al. 2012). The gripper is actuated by a brushed DC motor (Maxon-118797) driven by an H-bridge driver circuit (L298N) controlled via a dsPIC30F4011 microcontroller (Microchip), the system is shown in Fig. 1. For the experiments reported here we used feed-forward torque control.

\subsection{Working mechanism and its bioinspiration}

Hydrostatic skeletons are filled with liquid, usually water which has a high bulk modulus and hence resists significant volume changes. Contraction of muscle can decrease their relative dimensions; hence if circular, radial and transverse muscles are contracted, the diameter will decrease, if longitudinal muscles are contracted, the length will decrease. This dimensional change causes an increase of the internal pressure, and since no significant change in volume of the structure can occur, the decrease in one direction must result in an increase of the opposite (Kier 2012). The act of stiffening in constant volume structures like muscular hydrostats or more generally hydrostatic skeletons, occurs "if dimensional changes are resisted by either muscle activity or connective tissue" (Kier and Smith 1985). Kier and Smith (1985) proposed that "a structure wrapped with connective tissue which prevents dimensional change will stiffen in response to muscle contraction". Hence, after muscle contraction causes shortening in one dimension, the connective tissue resists the dimensional increase in the perpendicular direction and stiffening occurs. A similar resistance to dimensional change is observed in our gripper except that instead of connective tissue the gripper shell is made of a high tensile strength material. The working mechanism is the following: (i) pulling of the longitudinal wires decreases the gripper length. (ii) The shell material, initially loose because it is not fully filled with water, is put into tension, since no significant change in volume can occur and no dimensional increase in circumferential direction is possible. (iii) Hence the fluid pressure in the gripper rises, causing its stiffening.

Bending of the aforementioned constant volume structures can be produced by contraction of longitudinal muscles on one side of their body. Unilateral contraction will cause bending only if a constant diameter is maintained by applying a 
radial centripetal force, resisting the longitudinal compressional force, which tends to shorten the body. Without this resistance, there would be no bending but only shortening (Kier and Smith 1985). Similarly, in the gripper, the bending is initiated by the unilateral contraction of the longitudinal wires. However, in this case both bending and an element of shortening occur. The contraction of the longitudinal wires initially causes shortening of the gripper on one side while shifting the water to the opposite side. This increases the pressure of water on the internal walls of the gripper and the cross-section of the gripper goes from initially elliptical to circular. Any looseness of the wall disappears. Its high tensile strength opposes change of the gripper circumference and results in the bending of the gripper.

\subsection{Gripper variants}

Throughout the paper the two variants of the gripper, shown in Fig. 3, similar in every aspect but cable configuration, will be referred to as continuous gripper and discrete gripper. The first one has the same structure used in our previous work (Giannaccini et al. 2012) whereas the discrete one was specifically created to reduce friction between the cables and the tube and thus requires lower cable pulling forces. The difference between the two versions is in the cable system. In the continuous gripper the cable aiding the gripping motion is placed inside a single piece of continuous tube attached to the internal side of the outer layer. In the discrete gripper the cable aiding the gripping motion is placed within small equal lengths of tube (10 mm long) attached equidistant to each other on the internal side of the outer layer (the distance between the tubing pieces is $24 \mathrm{~mm}$ ). The performances of both grippers are compared in order to select the best one for stable grasping tasks. The evaluation will be carried out comparing the ability of the two grippers to achieve a large grasping workspace, which is directly related to the range of object size which can be grasped, and variable compliance. We were confident that friction

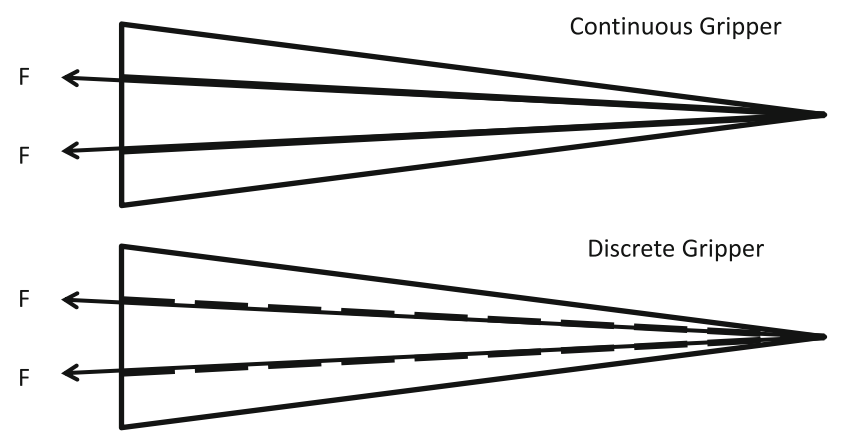

Fig. 3 Gripper variants: the first gripper has continuous tubing, shown by the solid lines and the second one has discrete tubing, shown by the dotted lines will be lower in the discrete gripper because the smaller tubing pieces offer a lower contact area and thus possibility of creating friction. This is confirmed by the experimental data; however, we also recorded a completely different curling behaviour for the second gripper, which was unexpected. Thus, conducting these experiments was necessary to fully characterise the grippers under real world conditions.

Firstly, the workspace of the two grippers was tested. This is an important measurement, which determines the grasping capabilities required for motion planning when employing the gripper as part of the complete robotic manipulator. Secondly, the two variants of the gripper were tested in order to assess the compliance variability within the applied force range. This is essential since, in the grasping task, the initial compliance allows conforming to the object shape, while in a later phase, a stable and more rigid configuration is more desirable. Once these issues were addressed, the ability of the best performing gripper to conform to object shape and provide multiple points of contact was tested. This experiment was conducted to demonstrate that the initial compliance is indeed well suited to increasing the contact area with the object, which reduces the local pressure the gripper exerts on it.

Finally, two experiments evaluating the performance within the specific application domain were conducted. In the first, it was demonstrated that the gripper could realise a stable grasp on a cup, an application specific object. When referring to a stable grasp, we refer to the definition by Feix et al. (2009) "A grasp is every static hand posture with which an object can be held securely with one hand". In our case, the gripper wrapped around the cup was able to securely suspend it in mid-air without slippage or fall of the cup, thus we defined that a stable grasp. In the second experiment, the gripper applied a stable grasp on an object freely positioned in space.

\section{Experimental analysis}

\subsection{Planar behaviour}

In this section we are taking into consideration the planar behaviour of the gripper, which can be compared to the bending movement in hydrostatic skeletons. In order to characterise the grippers' planar behaviour, i.e. the motion in the horizontal plane, their curvature profile and planar trajectory were analysed. The former was obtained by the radii of curvature as shown in Figs. 4 and 5. The experiments were conducted under increasing actuation forces on the cables. In addition, it was carried out an assessment of a variant's ability to best fit our requirement for a large workspace, under the minimum actuation $\left(F_{p}\right)$ forces. 


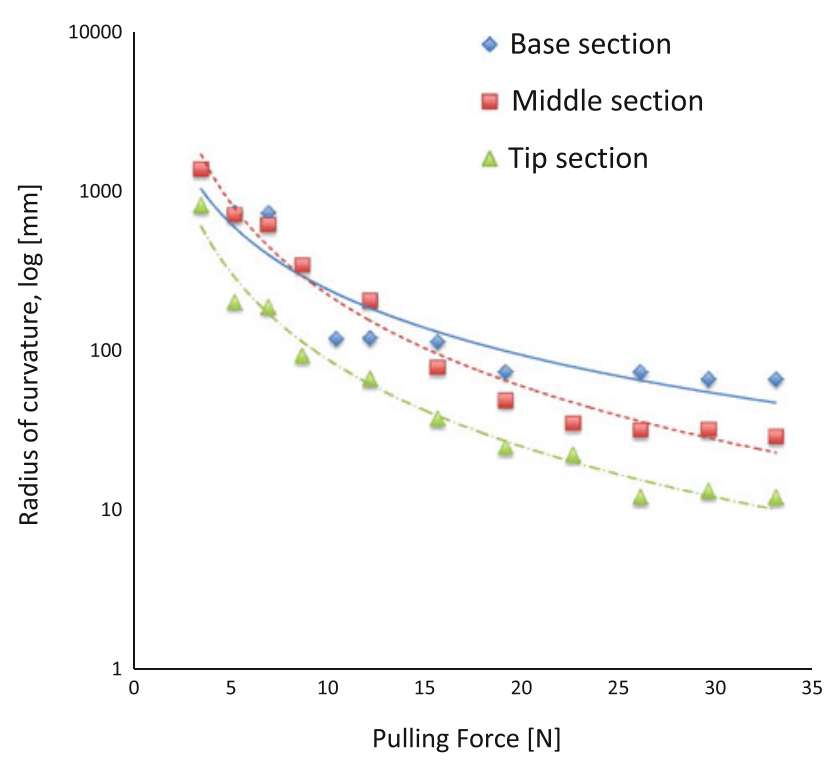

Fig. 4 Experimental variation of the radius of curvature in the continuous gripper. Please note that the $y$ axis has a logarithmic scale

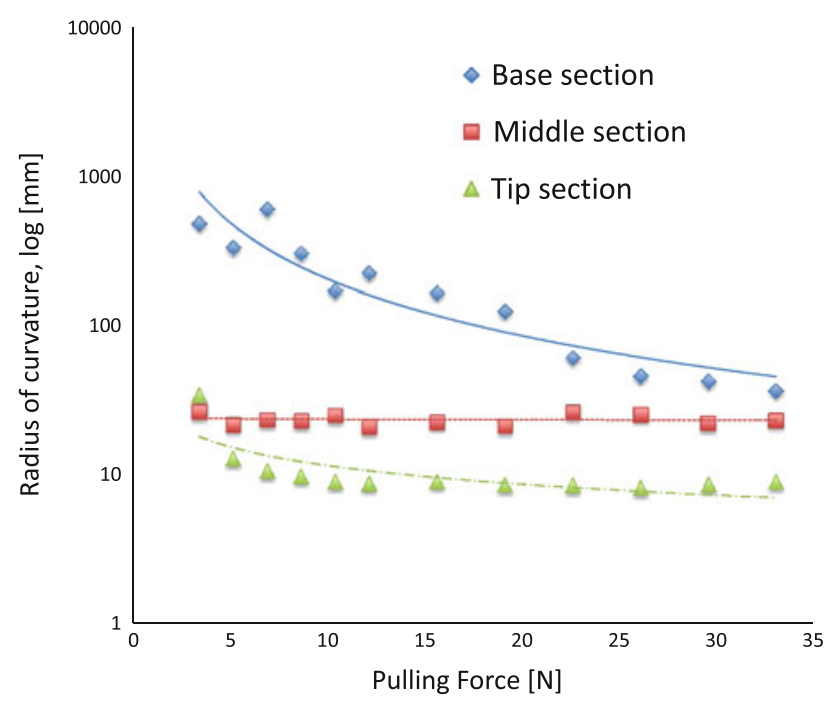

Fig. 5 Experimental variation of the radius of curvature in the discrete gripper. Please note that the $y$ axis has a logarithmic scale

\subsubsection{Experimental setup}

Nine equidistant markers were used to identify the different regions of the gripper. The markers were black dots drawn on the outer shell along its centreline. For the actuation, a force range, explicit in Fig. 6, was applied to the pulling cables. The markers on the gripper were tracked using a video camera and the footage was post-processed experimentally using tracking software (Robo-Realm ${ }^{\circledR}$ ). This allowed us to produce the $\mathrm{x}$ and $\mathrm{y}$ positions of the markers in the horizontal plane. These data were further processed with Matlab ${ }^{\circledR}$ to obtain the radius of curvature of each section concerned and the planar trajectory of the grippers. In order to calibrate the video images, the distance between the markers when the force on the cable was zero was used as a reference.

\subsubsection{Results}

Curvature profiles: Due to the nature of the gripper when 'curled up', fitting a single circle through all marker points was not possible. Hence, the decision to divide the gripper into 3 sections, with a respective circle each, shown in Fig. 7, was made during the post-processing phase. The circles fitted through the marker points of each sector, have an equation of the form

$(x-p)^{2}+(y-q)^{2}=r^{2}$

where $\mathrm{p}$ and $\mathrm{q}$ are the $\mathrm{x}$ and $\mathrm{y}$ coordinates of the centre of the circle and $r$ is the radius. Figures 4 and 5 show the results obtained for the continuous and discrete gripper respectively. In the initial position, when the $F_{p}$ is zero, all the markers are residing along the same line, thus, a circle cannot be defined. Hence, the initial position is not present in the data in Figs. 4 and 5 .

The graphs exhibit some important characteristics for both gripper configurations. Sections 1 and 2 of the continuous gripper, (the base and middle sections, see Fig. 7) show a similar bending trend with an increase in force exerted on the cables. Section 3 (the tip section) bends less when compared to the other two sections. The discrete gripper has shown different characteristics. The movement of section 1 is quite similar to the continuous gripper, however sections 2 and 3 do not show much movement above a $5 \mathrm{~N}$ pulling force. Sections 2 and 3 of the discrete gripper move first and then section 1 does, as opposed to the movement of the continuous gripper where the bending happens simultaneously along its length.

Planar trajectories: The planar trajectory results are shown in Fig. 6. The behaviour of the two grippers is clearly different, even under the same values of cable pulling forces and shows how a single change in the morphology (continuous or discrete cable tubing) causes a drastic change in the system behaviour. The initial force $(3.43 \mathrm{~N})$ barely makes the continuous gripper (Fig. 6a) move, while the discrete gripper (Fig. 6b) already starts curling. In the continuous gripper, the curvature in the gripper is minimal up to $12.13 \mathrm{~N}$. This is interesting for the grasping task since it allows the object to be grasped when distanced from the rigid holder. A further difference between the two gripper behaviours is that the tip of the discrete gripper bends even for small force values, while the continuous gripper curls simultaneously along all its sections. For this reason, the continuous gripper has a greater workspace. In Fig. 6a, relative to the continuous gripper, the maximum reach along the y axis is $180 \mathrm{~mm}$, while in Fig. 6b, relative to the discrete gripper, the maximum reach along the y axis is $70 \mathrm{~mm}$. On the contrary, 


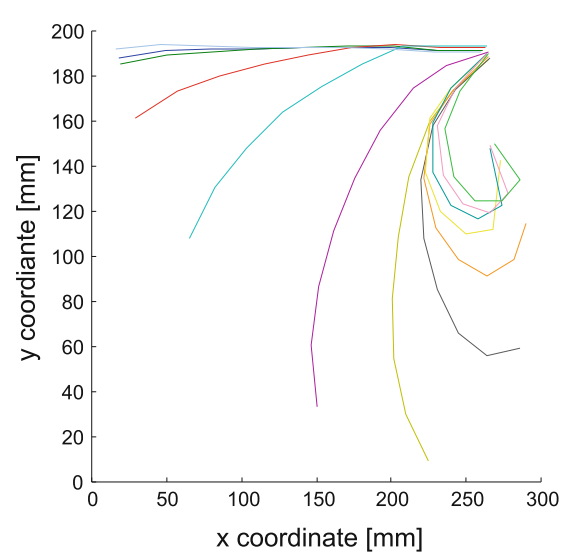

(a)

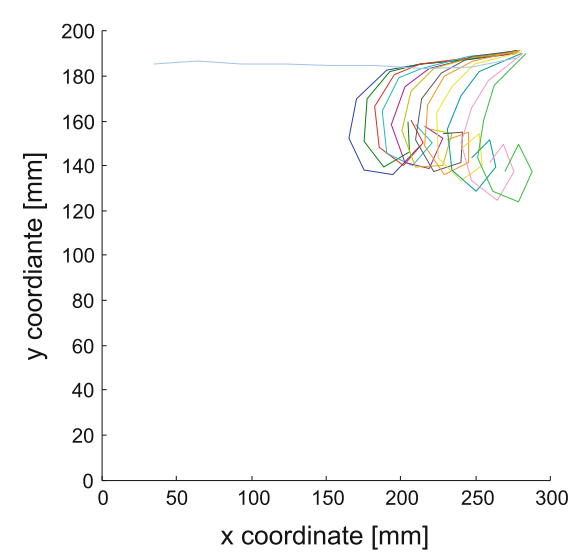

(b)

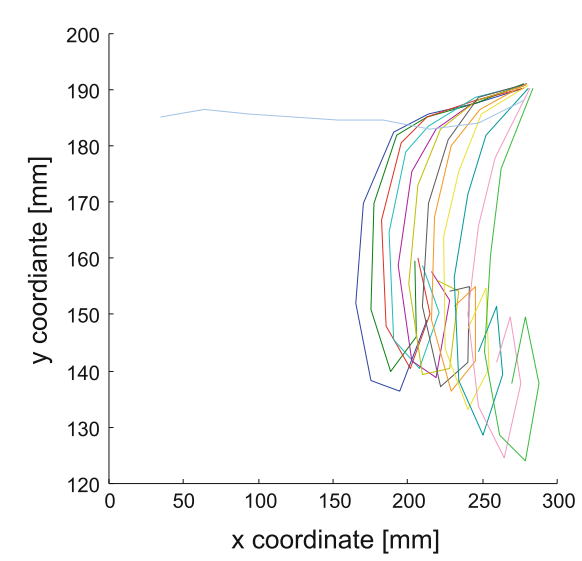

(c)
Fig. 6a Experimental trajectory on the horizontal plane: continuous gripper. b Experimental trajectory on the horizontal plane: discrete gripper. c Close up of the discrete gripper trajectory on the horizontal plane. The gripper holder is in position $x=280$ and $y=190$ in all three graphs. For all the three graphs the colour code matching the line to the pulling

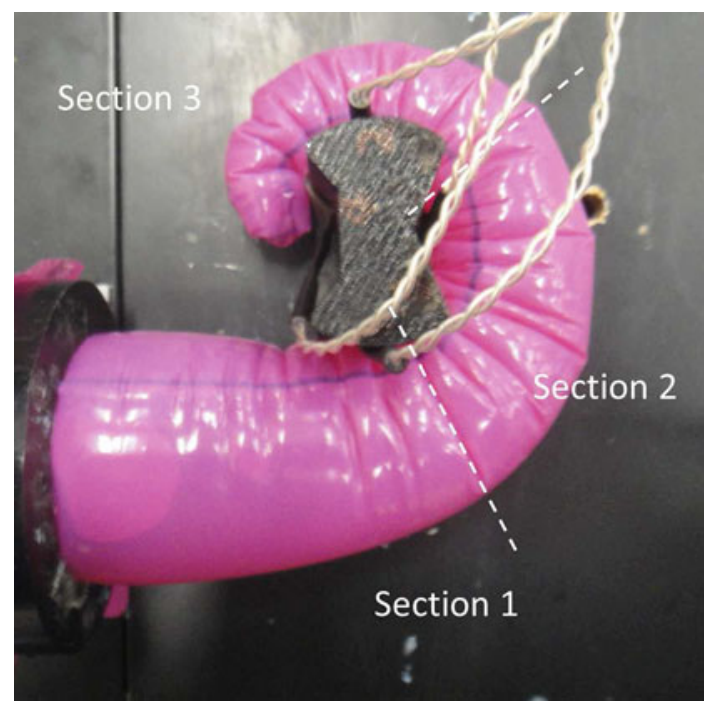

Fig. 7 The grippers are divided into sections: Section 1 corresponds to the base of the gripper, Section 2 to the middle and Section 3 to the tip. In the picture is possible to see also the twisted white wires of the pressure sensors, which are fixed on the object surface area. The internal side of the gripper is the one with the concertina and the external one is the one without folds

along the $\mathrm{x}$ axis, the maximum reach is the same for the two variants.

\subsection{Variable compliance}

The inspiration for the mechanism's variable compliance is provided by the stiffening movement in hydrostatic skeletons as described in Sect. 2.3. Compliance is the inverse of stiffness of a structure. Stiffness is the extent to which an object resists deformation once a force is applied to it. The change force applied to the cables in the rig is: Light blue: $0 \mathrm{~N}$, Blue: $3.43 \mathrm{~N}$, Dark green: $5.17 \mathrm{~N}$, Red: $6.91 \mathrm{~N}$, Turquoise: $8.65 \mathrm{~N}$, Purple: $10.39 \mathrm{~N}$, Light green: $12.13 \mathrm{~N}$, Black: $15.61 \mathrm{~N}$, Orange: $19.09 \mathrm{~N}$, Yellow: $22.57 \mathrm{~N}$, Petrol: 26.04N, Pink: 29.52N, Bright green: 33N (Color figure online)

in compliance in our gripper is measured as the change in stiffness.

In order to explain how the variable stiffness is achieved we are going to explain the gripper's working system. We define the potential volume, $V_{\text {pot }}$, of the shell as the maximum amount of water which the shell can hold: $170 \mathrm{ml}$. The potential volume depends on the folding of the structure. Thus, if the structure is partially folded, the potential volume is reduced. For example, if we were to fold the tip of the structure and then fill the structure with water, only part of the $170 \mathrm{ml}$ would fit in it.

We only fill the shell with $87 \%$ of the potential volume and remove all the air from the system. So, in this geometrical configuration, the current volume of the gripper (i.e. of the water), $V_{\text {curr }}$, is $87 \%(148 \mathrm{ml})$ of the potential volume of the unfolded gripper. This deflated structure is soft and compliant since the water can shift around in the shell. We call this state A. To increase the stiffness of the structure, we pull the cables, shown in Fig. 2, on the side of the gripper. By pulling the cables with a certain $F_{p}$, the material will first buckle then form folds along the side of the gripper where the cables are. This effect will cause a progressive shortening of the side of the gripper and a change in its geometry. In this new, partially folded, shape configuration the potential volume is reduced. The reduction of the potential volume of the shell continues until the potential volume matches the volume of the water. At this point, since water cannot be compressed, further pulling of the cables causes an increase of the pressure on the shell's walls, we call this state B. The higher pressure causes the increase in the stiffness of the gripper.

The mechanism of the stiffening is acting through the hoop stresses $(\sigma \theta)$ the material is exhibiting due to the increase in the shell-water system pressure. Given that the thickness of 
the shell $(\mathrm{t})$ is $0.06 \mathrm{~mm}$, thus smaller than the 1/10th of the radius (r) of the gripper ( $25 \mathrm{~mm}$ ), the simple form of YoungLaplace equation can be used to calculate hoop stresses:

$\sigma_{\theta}=\frac{P r}{t}$

Given that, $r$ and $t$ are effectively constant there is an increase of $\sigma_{\theta}$ proportional to the pressure $P . \sigma_{\theta}$ in all times must remain under the tensile strength of the material avoiding rupture. Moreover, $\sigma_{\theta}$ is resisting deformations perpendicular to the plane of the material. Given that stiffness is calculated by the amount of force applied over the deformation it creates

$k=\frac{F}{\delta}$

where $\mathrm{k}$ is the stiffness, $\mathrm{F}$ is the force applied on the body and $\delta$ is the displacement produced by the force along its direction of action. A smaller $\delta$ means a higher k. Hence, via the process of increasing pressure, leading to higher hoop stresses, countering deformation, a stiffness of the gripper is achieved.

The experiment described below has been conducted in order to demonstrate this stiffening. It is theorised that compliance decreases with the increase of force on the cables $\left(F_{p}\right)$, as the gripper bends.

\subsubsection{Experimental setup}

The apparatus used to estimate the structure stiffness is shown in Fig. 8. The horizontal platform is resting on the gripper's base in order to provide the compliance measurement in that

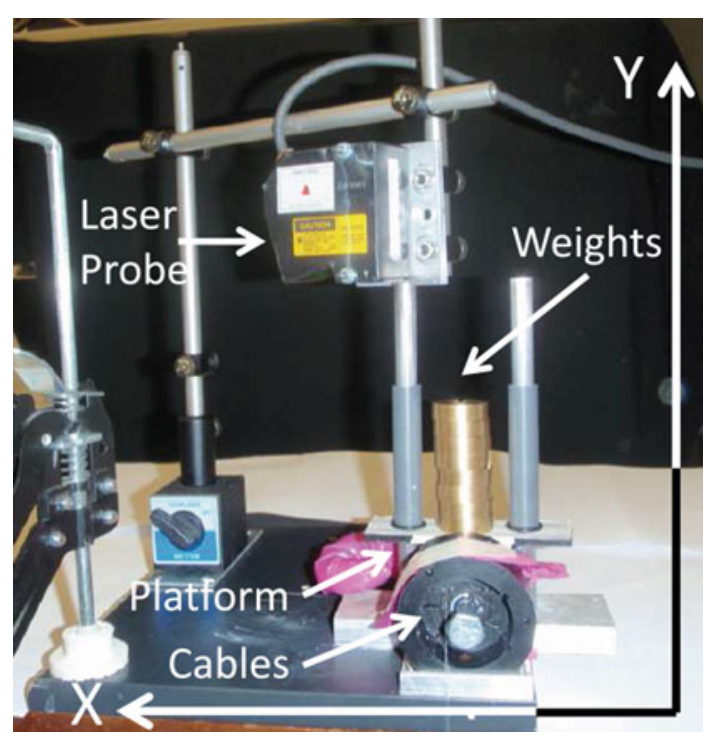

Fig. 8 Stiffness estimation setup. The laser probe is above the gripper. The displacement is along the $y$ direction, as shown in the figure coordinate system portion of the structure. During the experiment, an external force $F_{w}$ was applied on the gripper by adding weights (cylinders of $25 \mathrm{~mm}$ in diameter) on the platform. In order to obtain displacement $(\delta)$, a laser probe (LK-G152 and LK- GD500, Keyence) was used and $\delta$ is measured as a change in length (L) in the vertical (y axis) direction (see Fig. 8); horizontal displacement was not measured in this experiment.

\subsubsection{Results}

The experimental results can be seen in Fig. 9, showing the variation of the stiffness of the grippers at four different bending positions. These four positions correspond to 4 pulling force values $\left(F_{p}\right)$ for the cables $(0 \mathrm{~N}, 8.6 \mathrm{~N}, 15.6 \mathrm{~N}$ and $22.5 \mathrm{~N})$. These positions were chosen to allow investigation of the full spectrum of applicable forces. The results of the experiments show a change in the stiffness demonstrating the variable compliance of the gripper.

For ease of comparison, the same pulling forces $\left(F_{p}\right)$ were used in the stiffness estimation for the discrete gripper. This experiment was conducted with the same method as the experiment for the continuous gripper. The results of this experiment also show a change in the stiffness but a different behaviour at the same cable-pulling force values.

Once the values were obtained, we used a polynomial fit for the data. As can be seen in Fig. 9, the data for the continuous gripper is fitted in a second order polynomial (Eq. 4) and the data for the discrete gripper is fitted in a first order polynomial (Eq. 5). This function could be used to extrapolate the stiffness values for both gripper configurations. In Eqs. 4 and $5 \mathrm{~F}$ is in Newtons and $\mathrm{k}$ is in Newtons/millimetres.

$k_{c}(F)=a_{1,2} F_{1,2}^{2}+a_{1,1} F_{1,1}+a_{1,0}$
$k_{d}(F)=a_{2,1} F_{2,1}+a_{2,0}$

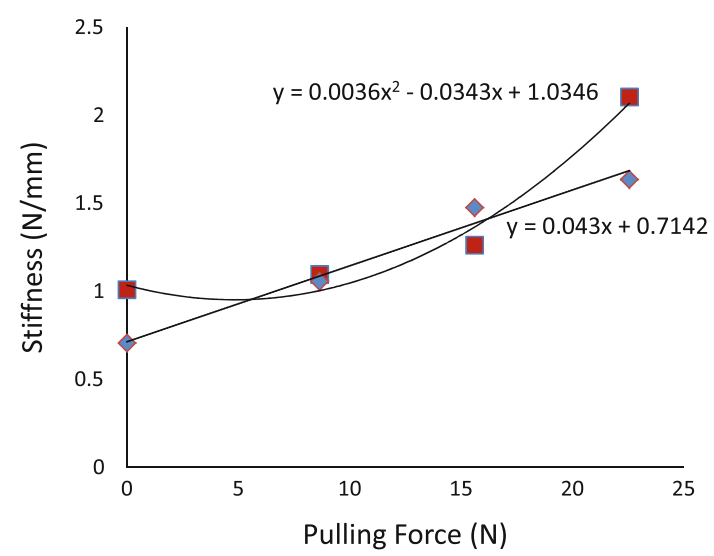

Fig. 9 Variation of the gripper stiffness, the diamonds represent the discrete gripper stiffness values, the squares represent the continuous gripper stiffness values. The experiment has been repeated multiple times and the values in the graph are the average values 
where $\mathrm{k}$ is the system stiffness, $\mathrm{F}$ is the force applied on the cables and $a_{x, y}$ are the constants: $a_{1,2}=0.0036$ $\frac{1}{\mathrm{~N}_{1} \mathrm{~mm}}, a_{1,1}=-0.0343 \frac{1}{\mathrm{~mm}}, a_{1,0}=1.0346 \frac{\mathrm{N}}{\mathrm{mm}}, a_{2,1}=0.043$ $\frac{1}{\mathrm{~mm}}, a_{2,0}=0.7142 \frac{\mathrm{N}}{\mathrm{mm}}$.

Given the restrictions due to the measuring rig, the full range of pulling forces could not be tested. These equations not only characterise the grasping system but could also be utilised by a higher-level control system. They provide the system designers with the important mapping between change in compliance and the applied pulling force $\left(F_{p}\right)$.

\subsection{Continuous over discrete Design}

The continuous gripper was chosen over the discrete gripper and all further experiments in this paper are conducted with the continuous gripper. The continuous gripper was chosen because of its greater workspace and ability to grasp a larger range of object sizes than the discrete gripper. Even if the discrete gripper presents lower friction, hence reducing the wasted energy in the system, it also presents numerous disadvantages.

Firstly, the discrete gripper has a reduced workspace, meaning the area it 'sweeps' during the closing motion, which is one of the main factors in determining the accuracy required to position the gripper in order to successfully grasp an object. Provided that the workspace is large, the positioning of the device is less of an issue even when using such a compliant gripper. Furthermore, the discrete gripper behaviour reduces the possible contact area with the object and this causes poor grasping capabilities. This is due to the fact that its tip curls up completely in the beginning of the grasp, as shown in Fig. 6b, which leaves only the base of the gripper available for grasping. Thus, the range of object sizes that can be picked up with the discrete gripper is smaller. The ability to grasp a large range of objects and to make up for the position inaccuracy inherent to this design are so important that we decided to discard the use of the discrete gripper. For these reasons, the continuous gripper was used in the practical experiments in the following section.

\subsection{Forward kinematics}

We aimed at establishing a kinematic model of the continuous gripper and we observed that its tip follows a spiral trajectory. Based on this, we conducted a number of experiments to evaluate this assumption and establish a connection between the displacement of the actuating string to the location of the tip, hence the forward kinematics of the gripper. The general equation, in polar coordinates, for spirals is given as:

$r=a \cdot \theta^{\frac{1}{n}}$ where, $a$ and $n$ are parameters defining the size and winding of the spiral respectively. The simplest form of spiral is the Archimedes' spiral with parameter $n=1$, thus, a linear relationship between $\theta$ and $r$.

Polar coordinates can be expressed in cartesian ones simply via the equations:

$x=r \cdot \cos (\theta)$

$y=r \cdot \sin (\theta)$

and by combining (6) and (7) a connection between $x, y$ and $\theta$ can be derived.

$x=a \cdot \theta \cdot \cos (\theta)$

$y=a \cdot \theta \cdot \sin (\theta)$

To evaluate Eq. 8 we conducted a set of experiments (six in number) where we increased the displacement of the wires $d$ by specific steps (i.e. $10 \mathrm{~mm}$ ) and recorded, using a vision tracking system, the $x$ and $y$ coordinates of the end-effector. Then, using the curve fitting toolbox in Matlab ${ }^{\circledR}$, we fitted slightly varied Eq. 8 to the data. Specifically the varied equations were:

$x=a_{x} \cdot \theta \cdot \sin \left(b_{x} \cdot \theta+\pi / 2\right)+c_{x}$

$y=a_{y} \cdot \theta \cdot \sin \left(b_{y} \cdot \theta\right)+c_{y}$

The fitting process provided us with the parameters that would allow us to establish the forward kinematic model of the gripper. Also, polar coordinate angle $\theta$ must be related to the actuation parameter, the displacement $d$ of the wires. By observation, the spiral-like shape of the gripper has 1.5 turns when fully closed, while the maximum displacement of the wires is $140 \mathrm{~mm}$.

It must be noted that a spiral described by Eq. 6 is considered starting in the 'tip', while we are presenting it here from the base of the griper which is the spiral point at $\theta=1.5 \cdot \pi$. Hence, in order to align the observed data and the model equation the relation between $\theta$ and displacement $d$ is given as a decrease of the displacement:

$\theta=\frac{1.5 \cdot \pi}{140} \cdot(140-d) \Rightarrow \theta=0.034 \cdot(140-d)$

The results of the fitting process can be seen in Fig. 10. Also the statistical details regarding the definition of the parameters can be seen in Table 1 . The $R^{2}$ for the fitting of coordinate $x$ to $(9 a)$ is 0.985 , and for coordinate $y$ to (9b) is 0.886 . Both values indicate that the respective fitting process gave a good result. Finally the forward kinematics equations for the end-effectors are: 


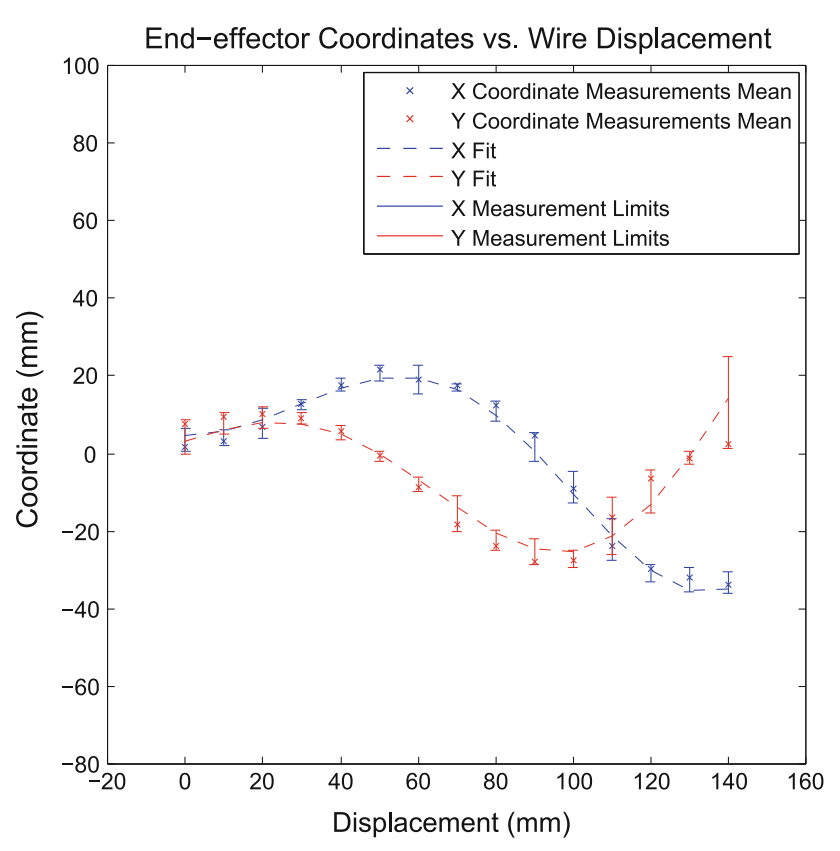

Fig. 10 Measurements for the end-effector. The mean value among the six tests for each coordinate over the full displacement range is given. Also, the limits for each displacement and the fitted curve are depicted

Table 1 Parameters fitting statistic analysis

\begin{tabular}{lrccl}
\hline Parameter & Value & $95 \%$ low limit & $95 \%$ high limit & $\begin{array}{l}\text { Standard } \\
\text { deviation }\end{array}$ \\
\hline$a_{x}$ & 12.775 & 11.787 & 13.757 & 0.548 \\
$b_{x}$ & 1.075 & 1.040 & 1.109 & 0.026 \\
$c_{x}$ & 6.490 & 4.008 & 9.972 & 0.988 \\
$a_{y}$ & 13.070 & 10.171 & 15.976 & 1.119 \\
$b_{y}$ & 1.045 & 1.006 & 1.084 & 0.014 \\
$c_{y}$ & 4.514 & -0.749 & 9.837 & 1.242 \\
\hline
\end{tabular}

$$
\begin{aligned}
x= & 12.775 \cdot 0.034 \cdot d \cdot \sin (1.075 \cdot 0.034 \cdot d+\pi / 2) \\
& +6.490 \\
y= & 13.070 \cdot 0.034 \cdot d \cdot \sin (1.045 \cdot 0.034 \cdot d) \\
& +4.514
\end{aligned}
$$

\subsection{Variable cross-section shape grasping}

The aim of this experiment is to show that the compliant and continuum physical structure of the gripper adapts to the shape of the object without previous knowledge of it (Variable cross-section shape grasping). For this reason, three objects with different cross sections were grasped using the same actuation forces.
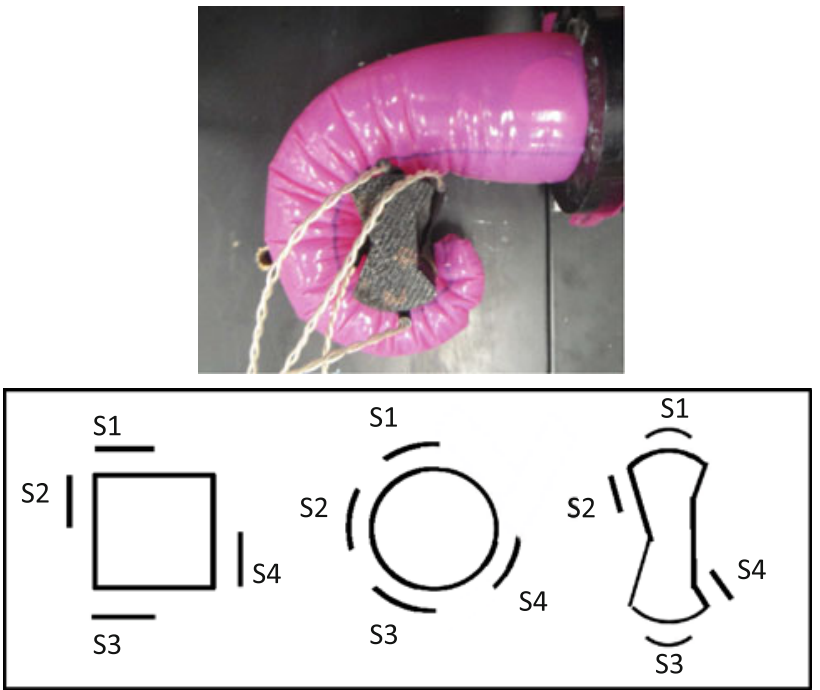

Fig. 11 Photo: the continuous gripper as it is grasping the irregular object. Schematics: Position of the sensors on the tested objects, $S$ stands for sensor: $S 1$ represents the first sensor, $S 2$ the second sensor and so on. The orientation of the object in the schematics is the same as the orientation of the object in the picture. From the left: square, round and irregular object

\subsubsection{Experimental setup}

The arrangement of the experimental setup is shown in Fig. 11. The gripper holder was fixed on a board and the maximum applicable force $(34.3 \mathrm{~N})$ was applied in order to grasp the objects. This force was chosen because preliminary experiments showed that it ensured that the object was fully encircled and grasped firmly. The objects were fixed to the table surface.

Since the chosen objects have curved surfaces, the tactile sensor (Contact 500, Pressure Profile System, Inc) was chosen for its size and flexibility, and a NI-DAQ (National Instrument) board interfaced with a PC was used to obtain the sensors' measurements. LabView SignalExpress software ${ }^{\circledR}$ was used to analyse the output data received over the NI-DAQ board. Four sensors were fixed to the external surface of each object. The positions at which the sensors were placed are illustrated in Fig. 11.

\subsubsection{Results}

The gripper's passive ability to comply with objects' shape was tested for each object. Each experiment was repeated five times in order to ensure its repeatability and every time the same number of sensors have been touched. The change in force displayed by the sensors mounted on the object indicates whether it was touched by the gripper.

As seen in Fig. 12, in all cases, multiple changes in force applied to the sensors are obtained. In the first and second 

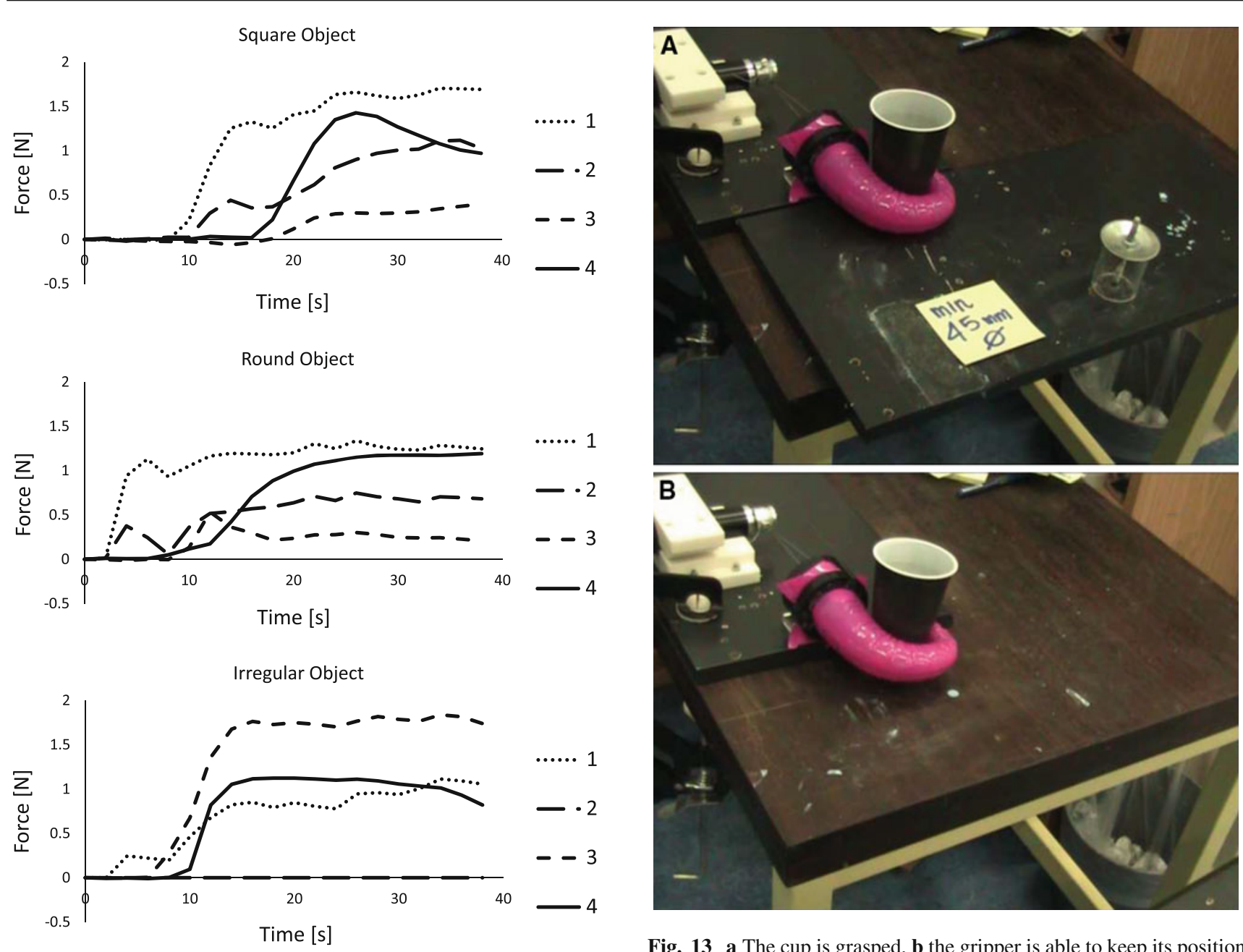

Fig. 12 Force readings of the four sensors on the square, round and irregular object

experiment, all sensors produce an increased output, in the third, three out of four sensors display a change in force.

The gripper is able to mould to the oval shape of the irregular object (demonstrated by the fact that Sensors 3 and 4 have show an increase in force). However, it does not trigger Sensor 2 because of its bending trajectory and the object's intrinsic geometry.

\section{Experiments in the application domain}

To focus on a specific application domain, we chose grasping of an everyday object such as a cup, because it is a very common task. Also, it is important to show that the gripper initial compliance is not a hindrance in free space grasping. The two following experiments have been conducted with the continuous gripper, the best performing of the two variants we tested.

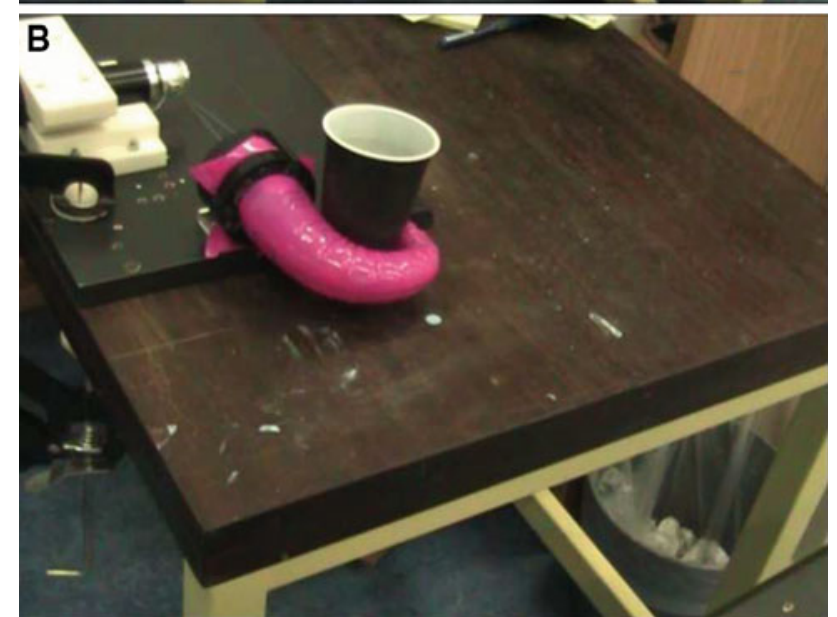

Fig. 13 a The cup is grasped, $\mathbf{b}$ the gripper is able to keep its position without needing the extra support

\subsection{Grasping and holding a cup}

The following experiment focuses on the ability of the gripper not only to grasp but also to hold a plastic cup. The cup, which has a minimum diameter of $45 \mathrm{~mm}$, is placed on a removable slate, Fig. 13. Subsequently, the gripper cables are driven with the maximum applicable force $(34.3 \mathrm{~N})$, in order to keep consistency with the experiments in Sect. 3. Once the partially filled cup $(0.065 \mathrm{~kg})$ was grasped, the slate underneath the cup was removed. It was observed that the cup remained in place and did not tilt. This demonstrates both the stability of the grasp and the purely horizontal grasping motion with no torsional forces applied to the object. In the specific example of the cup, this last characteristic is vital since it ensures that a liquid contained in the cup would not get spilled. Furthermore, the cup is not deformed by the grasp either, due to the large contact area provided by the gripper. This characteristic facilitates sensible handing-over of fragile objects. 

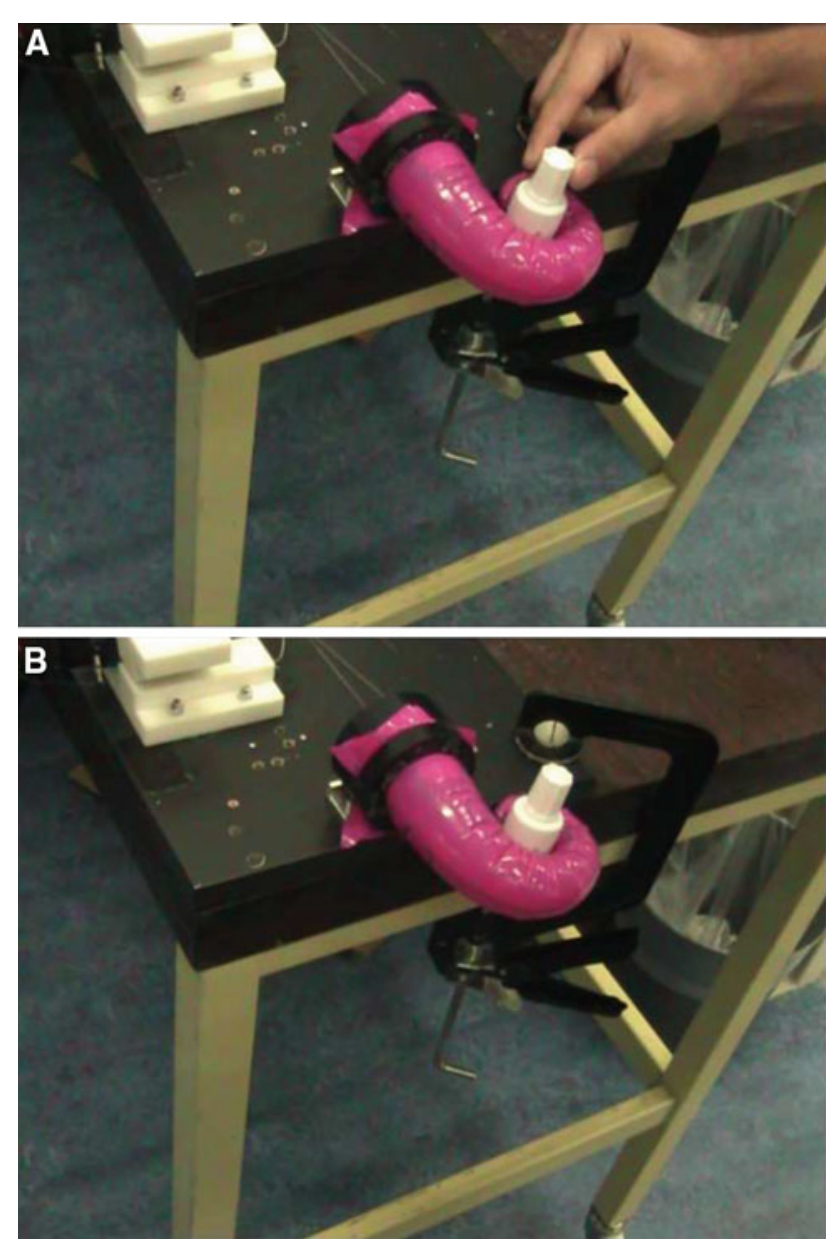

Fig. 14 Grasping in free space. a The object is held by the human, b the gripper has successfully grasped the object

\subsection{Free space grasping}

The following experiment aims to prove that the grasping and holding tasks can also be performed in free space. Thus, the gripper support is held at the edge of the table and the gripper is hanging in mid-air. Previously, the experiments were conducted with both gripper and object resting on a surface.

An object is held close to the gripper by a person. The robotic system is activated and the gripper starts curling and levelling itself on the horizontal plane. The object is successfully grasped, then released by the person but held in place by the gripper (Fig. 14).

\section{Discussion}

The described experiments demonstrated the versatility of the proposed gripper to achieve a stable grasping action under uncertain conditions.
The overall results of the planar behaviour experiments indicate that the continuous gripper features a greater workspace than the discrete gripper. For this reason, the continuous gripper is chosen as the better performing of the two variants. The confirmation of the similarity between the bending movement in hydrostatic skeletons and in our device, compared in Sect. 2.3, can be found in the graphs of the gripper planar trajectory in Fig. 6a. Although, substantial bending of the structure starts only after the initial phase of shortening of the gripper.

Similarly, the mechanism that causes stiffening in hydrostatic skeletons is successfully reproduced in the gripper design, as demonstrated by the increase in stiffness shown in Fig. 9. Variable compliance is present in both gripper variants. It changes linearly in the discrete gripper while the continuous gripper achieves the same stiffness end value but the change in the stiffness is described by a second order equation. This is due to the difference in cable structure which yields a different curling behaviour and water displacement.

The results of the compliance to the object experiments demonstrate that more points of contact are achieved when compared, for example, to the conventional rigid clamp gripper in SCHUNK (2013), where the pressure points are normally limited to two areas. In case a multi-fingered gripper (e.g. an anthropomorphic hand) was used to perform a lateral grasp on roughly cylindrical objects with very different cross-sections, each finger would need to be individually controlled in order to obtain similar results. Hence, the absence of rigid or semi-rigid structures ensures that, in imitating a lateral grasp (or wrap) primitive, our design can adapt to different shapes passively, greatly simplifying control and the mechanical design.

The gripper's compliance is good to avoid crushing the object since it creates a higher contact area than rigid grippers. Furthermore, due to its initially very compliant structure our gripper is more adaptable to the object's cross-section shape compared to traditional grippers. In addition, only simple grasp planning is required, thus little computational effort is necessary.

\section{Conclusion and future work}

In this work, it has been shown that a novel gripper with very simple, inexpensive structure can effectively grasp and hold roughly cylindrical objects of very different cross-sectional shapes. This is achieved with a very simple control mechanism and its extremely compliant structure which allows it to successfully conform to these objects. Unlike many continuum robots which are made of multiple compliant sections joint by rigid plates (Hannan and Walker 2003), our structure is a fully continuous one, without any disruptions or rigid elements. This characteristic, inspired by hydrostatic skeletons, 
ensures adaptability to the object. It has been further demonstrated that the gripper's compliance varies ensuring both a compliant grasp and a stable hold. This ability validates that our gripper is not only a novel and interesting concept but that it could be successfully employed in everyday tasks.

Furthermore it was not necessary to employ advanced, multi dimensional control algorithms to achieve the grasp and holding task. No on-line adjustment of the motor control parameters was necessary and feed-forward control sufficed to actuate the gripper. This feature is due to the gripper's unique physical structure. Passive structures usually suffer from a limited behaviour, however, the change in material properties brought about by the interaction between the water and the shell in our device remediates this shortcoming by passively coupling the appropriate degree of stiffness to each phase of the grasping task.

The work in progress includes the identification of the gripper's trajectories during free space object grasping. Performance can be improved by studying the employment of such materials as composites or smart materials. The addition of sensors to the system is under consideration, to further improve the ability of the gripper to interact with the environment. Furthermore, to ensure that every direction of the passive compliance is exploited to aid the grasping action, the number of cables in the gripper will be increased to six. Thus, the gripper will be able to grasp in any orientation in space exploiting fully its continuum and omnidirectional compliance. The gripper will be attached to a manipulator which also features variable compliance. The system is under construction and intended to work in a human-occupied environment.

Acknowledgments We would like to thank Yue Zheng, Peng Huang and Dr Peter Walters for their excellent hardware support, help in conducting the experiments and fruitful discussions about the prototype. This work has been funded by the European Commission. It is part of the INTRO (INTeractive RObotics Research Network) project, in the Marie Curie Initial Training Networks (ITN) framework, Grant Agreement No.: 238486

\section{References}

Brown, E., Rodenberg, N., Amend, J., Mozeika, A., Steltz, E., Zakin, M. R., et al. (2010). Universal robotic gripper based on the jamming of granular material. Proceedings of the National Academy of Sciences, 107(44), 18809-18814.

Calisti, M., Arienti, A., Giannaccini, M. E., Follador, M., Giorelli, M., Cianchetti, M., Mazzolai, B., Laschi, C., \& Dario P. Study and fabrication of bioinspired octopus arm mockups tested on a multipurpose platform. In 20103 rd IEEE RAS and EMBS International Conference on Biomedical Robotics and Biomechatronics (BioRob) (pp. 461-466).

Cheng, N. G., Lobovsky, M. B., Keating, S. J., Setapen, A. M., Gero, K. I., Hosoi, A. E., \& Iagnemma, K. D. (2012). Design and analysis of a robust, low-cost, highly articulated manipulator enabled by jamming of granular media. In 2012 IEEE International Conference on Robotics and Automation (ICRA) (pp. 4328-4333).
Deimel, R., \& Brock, O. (2013). A compliant hand based on a novel pneumatic actuator. In 2013 IEEE International Conference on Robotics and Automation (ICRA) (pp. 2039-2045).

Dollar, A. M., \& Howe, R. D. (2010). The highly adaptive SDM hand: Design and performance evaluation. The International Journal of Robotics Research, 29(5), 585-597.

Eppner, C., Bartels, G., \& Brock, O. (2012). A compliance-centric view of grasping. Technical Report.

Feix, T., Pawlik, R., Schmiedmayer, H., Romero, J., \& Kragic, D. (2009). A comprehensive grasp taxonomy. In Robotics, Science and Systems: Workshop on Understanding the Human Hand for Advancing Robotic Manipulation (pp. 2-3).

FESTO. Retireved February 26, 2013, from http://www.festo.com/rep/ encorp/assets/pdf/BHAen.phf.

Giannaccini, M., Dogramadzi, S., \& Pipe, T. (2011). Solutions for a variable compliance gripper design. In Towards Autonomous Robotic Systems. Lecture Note in Computer Science (Vol. 6856, pp. 410 411).

Giannaccini, M. E., Zheng, Y., Dogramadzi, S., \& Pipe, T. (2012). Towards a variable compliance hydrostatic skeleton inspired gripper. In 2012 4th IEEE RAS and EMBS International Conference onBiomedical Robotics and Biomechatronics (BioRob) (pp. 246-251).

Grebenstein, M., Albu-Schaffer, A., Bahls, T., Chalon, M., Eiberger, O., Friedl, W., Gruber, R. et al. (2011). The dlr hand arm system. In 2011 IEEE International Conference on Robotics and Automation (ICRA) (pp. 3175-3182).

Hannan, M. W., \& Walker, I. D. (2003). Kinematics and the implementation of an elephant's trunk manipulator and other continuum style robots. Journal of Robotic Systems, 20(2), 45-63.

Hirose, S., \& Umetani, Y. (1978). The development of soft gripper for the versatile robot hand. Mechanism and Machine Theory, 13(3), 351-359.

Ilievski, F., Mazzeo, A. D., Shepherd, R. F., Chen, X., \& Whitesides, G. M. (2011). Soft robotics for chemists. Angewandte Chemie, 123(8), 1930-1935.

INTRO project. Retrieved February 27, 2013, from http://introbotics. $\mathrm{eu} /$.

Kier, W. M. (2012). The diversity of hydrostatic skeletons. The Journal of Experimental Biology, 215(8), 1247-1257.

Kier, W. M., \& Smith, K. K. (1985). Tongues, tentacles and trunks: The biomechanics of movement in muscular-hydrostats. Zoological Journal of the Linnean Society, 83(4), 307-324.

McMahan, W., Chitrakaran, V., Csencsits, M., Dawson, D., Walker, I. D., Jones, B., Pritts, A. M., Dienno, Grissom, D. M., \& Rahn, C. D. (2006). Field trials and testing of the OctArm continuum manipulator. In Proceedings of 2006 IEEE International Conference on Robotics and Automation (ICRA 2006) (pp. 2336-2341).

Menciassi, A., Accoto, D., Gorini, S., \& Dario, P. (2006). Development of a biomimetic miniature robotic crawler. Autonomous Robots, 21(2), 155-163.

Pfeifer, R., Lungarella, M., \& Iida, F. (2007). Self-organization, embodiment, and biologically inspired robotics. Science, 318(5853), 10881093.

SCHUNK. Retrieved January 30, 2013, from http://www.gb.schunk. com/schunk/schunk_websites/products/products.html?product level_1=244\&product_level_2=250\&product_level_3=0\&\& country $=$ GBR $\& \operatorname{lng}$ Code $=E N \& \operatorname{lng}$ Code $2=$ EN.

SHADOW. Retrieved February 26, 2013, from http://www. shadowrobot.com/hand/motorhand.shtml.

Taylor, J. R., \& Kier, W. M. (2003). Switching skeletons: Hydrostatic support in molting crabs. Science, 301(5630), 209-210.

Trimmer, B. A., Takesian, A., Sweet, B., Rogers, C. B., Hake, D. C., \& Rogers, D. J. (2006). Caterpillar locomotion: A new model for softbodied climbing and burrowing robots. In 7th International Symposium on Technology and the Mine Problem (Vol. 1, pp. 1-10). Monterey, CA: Mine Warfare Association. 
Vaidyanathan, R., Chiel, H. J., \& Quinn, R. D. (2000). A hydrostatic robot for marine applications. Robotics and Autonomous Systems, $30(1), 103-113$.

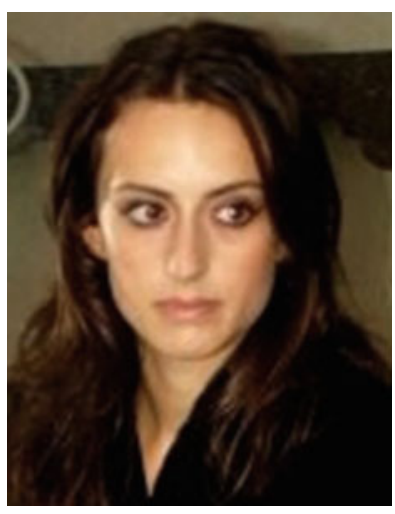

M. E. Giannaccini gained a Bachelor and a Masters degree (cum laude) in Biomedical Engineering at the University of Pisa, Italy and worked on her Master dissertation on the control of an octopus-like soft arm in Scuola Superiore Sant'Anna, Italy. She is a Marie Curie fellow in the EU funded ITN project INTRO and a $\mathrm{PhD}$ student at the Bristol Robotics Laboratory, UK. INTRO aims to create technologies needed to build intelligent robots that function in close interaction with humans in unstructured, chang-

ing real word conditions

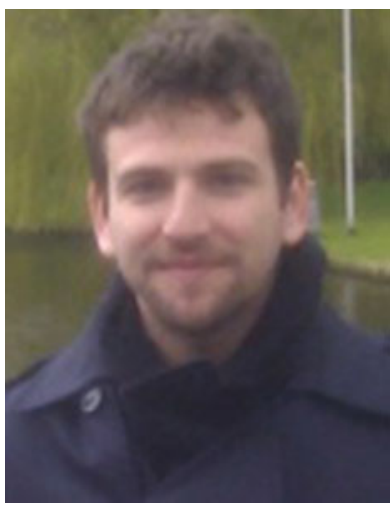

I. Georgilas received the Dipl. in Production Engineering (5years course) and Ph.D. degree in Production Systems from Democritus University of Thrace (DUTH) at the Department of Production Engineering and Management. From June 2010 to January 2011 he was an Assistant Researcher at the Department of Production Engineering and Management, School of Engineering, Democritus University of Thrace. Currently he is a Research Associate at the Bristol Robotics Laboratory, Bristol. His research interests include the development of mechatronic systems with focus on the mechanical design and implementation of biologically inspired solutions, both for academic and industrial applications.

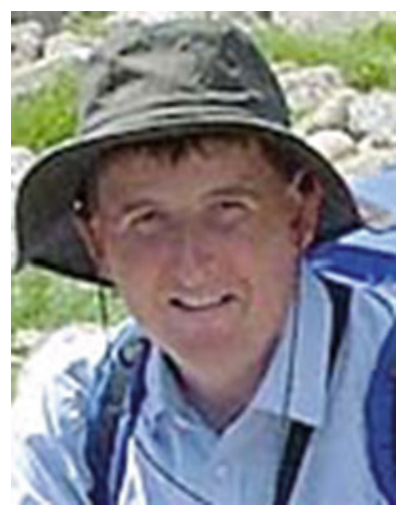

I. Horsfield graduated from Kent University in 1985 with an honours Degree in Electronic Engineering to join Rolls Royce at Bristol in the Industrial Electronics and Automation support team. In 1993 I moved to The University of the West of England to be a member of the IAS lab. I have been responsible for designing and building a number of major projects in the lab including U-Bot fleet, Grey Walter Turtles, Millennium Dome robots, Submar fleet and Ecobot series. I have also contributed to a number of papers and other projects including the Linuxbot fleet.

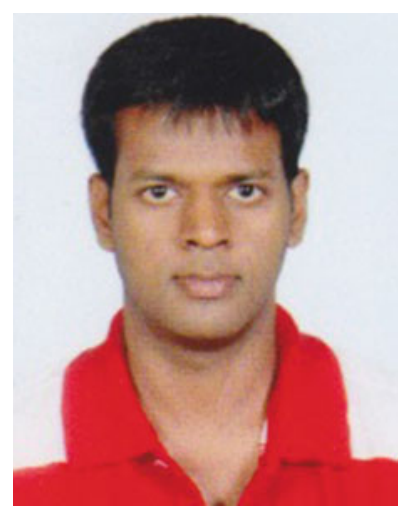

B. H. P. M. Peiris born in 1989, I am a citizen of Sri Lanka. I obtained my bachelors (B.E.) in Mechanical Engineering from the University of Bristol in 2011. Robotics research being an integral part of my university undergraduate career, I followed an MSc in Advance Robotics Engineering from the same university soon afterwards. My research interests have been in biologically inspired robotics where I've been actively been involved in research with Electro-Active Polymer technology.

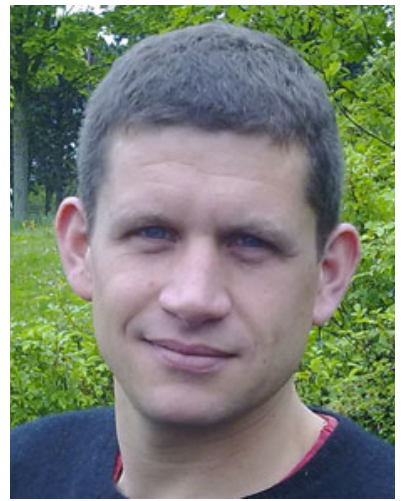

A. Lenz received a Microelectronics Engineering Degree from the University of Applied Sciences, Trier, Germany, in 2001, and the M. Sc. Degree in advanced technologies in electronics from the University of the West of England, Bristol, U.K., in 2002. He received the $\mathrm{Ph} . \mathrm{D}$. Degree from the University of the West of England, in 2011, investigating cerebellum inspired robotic gaze control. $\mathrm{He}$ has worked for Siemens VDO, Germany, as an Embedded Software Engineer for the automotive industry and is currently a Research Fellow with the Bristol Robotics Laboratory, Bristol, addressing embedded control and collaboration issues in bioinspired systems and human-robot cooperation. His research interests include distributed real-time systems, embedded sensing and control, robotics, and bioinspiration.

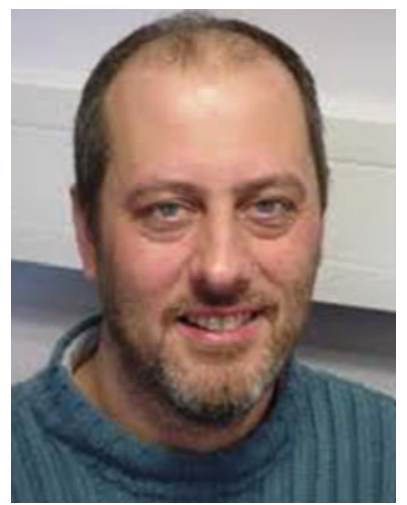

A. G. Pipe Deputy Director of the Bristol Robotics Laboratory, a Reader in Robotics \& Autonomous Systems at UWE and a visiting Research Fellow at the University of Bristol. He has 15 years of research experience in advanced robotics, machine learning and adaptive behaviour applied to intelligent \& distributed control/monitoring systems, and in embedding complex algorithms (such as biologicallyplausible spiking neural networks) in reprogrammable digital VLSI. He has worked extensively on adaptive joint-controllers for multiaxis robot manipulators, where the focus has been on proving convergence during adaptation and stability of the overall system using Lyapunov theory, and on efficient reaching strategies for redundant robot manipulators. His research work has resulted in over 80 publications in international refereed journals and conferences. Four awarded research grants since 2004 come to a total of $£ 1.7 \mathrm{M}$. Tony also recently joined the BSI/ISO standardisation initiative, 'Robots for Personal Care. 


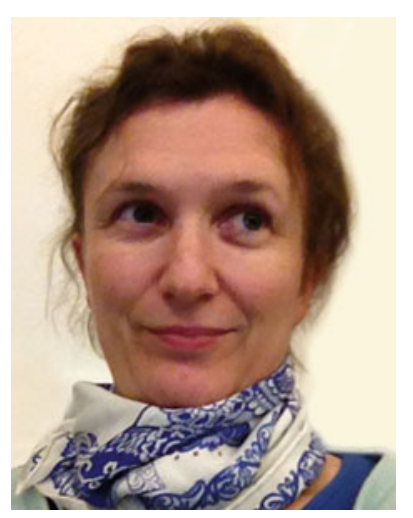

S. Dogramadzi Associate Professor in Robotics. She has more than 14 years of experience in academy doing research in engineering related topics including medical engineering, control applications and robotics. Her main achievements include: Being awarded research grants from the EC and EPSRC, promoting research through refereed publications and media coverage, co-chairing IEEE Conferences, organizing and taking part in robotics events and public discussions about robotics, taking active part in the ISO TC184/S2 responsible for development of Service robots standards and the JWG9 (Joint Working Group between the ISO and IEC), recently established to develop standards for Medical Robotics. She is a project manager for several EC and the EPSRC projects and coordinates the work between partners and researchers. Apart from regular teaching duties, she holds several administrative positions at the University including the programme leader of master courses in Robotics. SD leads a Medical Robotics group in Bristol Robotics Laboratory. 